\title{
An evolvable space telescope for future astronomical missions
}

Ronald S. Polidan, James B. Breckinridge, Charles F. Lillie, Howard A. MacEwen, Martin R. Flannery, et al.

Ronald S. Polidan, James B. Breckinridge, Charles F. Lillie, Howard A. MacEwen, Martin R. Flannery, Dean R Dailey, "An evolvable space telescope for future astronomical missions," Proc. SPIE 9143, Space Telescopes and Instrumentation 2014: Optical, Infrared, and Millimeter Wave, 914319 (2 August 2014); doi: 10.1117/12.2057161

Event: SPIE Astronomical Telescopes + Instrumentation, 2014, Montréal, Quebec, Canada 


\title{
An Evolvable Space Telescope for Future Astronomical Missions
}

\author{
Ronald S Polidan $^{* a}$ James B Breckinridge $^{\mathrm{b}}$, Charles F Lillie ${ }^{\mathrm{c}}$, Howard A MacEwen ${ }^{\mathrm{d}}$, \\ Martin R Flannery ${ }^{\mathrm{a}}$ and Dean R Dailey ${ }^{\mathrm{a}}$ \\ ${ }^{a}$ Northrop Grumman Aerospace Systems, One Space Park Dr., Redondo Beach, CA 90278 \\ ${ }^{\mathrm{b}}$ Breckinridge Associates, LLC, 985 East California Boulevard, Pasadena, CA. 91106 \\ 'Lillie Consulting LLC, 6202 Vista del Mar, Playa del Rey, CA 90293 \\ ${ }^{\mathrm{d}}$ Reviresco LLC, 4901 Loosestrife Court, Annandale, VA 22003
}

\begin{abstract}
Astronomical flagship missions after JWST will require affordable space telescopes and science instruments. Innovative spacecraft-electro-opto-mechanical system architectures matched to the science requirements are needed for observations for exoplanet characterization, cosmology, dark energy, galactic evolution formation of stars and planets, and many other research areas. The needs and requirements to perform this science will continue to drive us toward larger and larger apertures. Recent technology developments in precision station keeping of spacecraft, interplanetary transfer orbits, wavefront/sensing and control, laser engineering, macroscopic application of nano-technology, lossless optical designs, deployed structures, thermal management, interferometry, detectors and signal processing enable innovative telescope/system architectures with break-through performance. Unfortunately, NASA's budget for Astrophysics is unlikely to be able to support the funding required for the $8 \mathrm{~m}$ to $16 \mathrm{~m}$ telescopes that have been studied as a follow-on to JWST using similar development/assembly approaches without decimating the rest of the Astrophysics Division's budget. Consequently, we have been examining the feasibility of developing an "Evolvable Space Telescope" that would begin as a 3 to $4 \mathrm{~m}$ telescope when placed on orbit and then periodically be augmented with additional mirror segments, structures, and newer instruments to evolve the telescope and achieve the performance of a $16 \mathrm{~m}$ or larger space telescope. This paper reviews the approach for such a mission and identifies and discusses candidate architectures.
\end{abstract}

Keywords: Space telescope, segmented telescopes, in-space assembly and servicing

\section{INTRODUCTION}

The Hubble Space Telescope (HST) has for decades provided some of the most valuable insights into the science of astronomy and astrophysics. Similarly the other great astronomical observatories of the past, Chandra, Spitzer, and Compton have contributed immensely to advancing science and our general knowledge of the universe. These large observatories are the some of the most productive "science machines" ever built. This tradition is expected to continue with the James Webb Space Telescope (JWST) when it is launched in 2018. Astrophysics, whether space based or ground based, is continuously driven to larger and larger apertures in order to explore the distant universe or study faint nearby objects such as exoplanets.

Unfortunately but not unexpectedly, these great observatories have also been some of the most costly observatories built by NASA. All of these observatories were threatened with cancellation during development due to cost and schedule overruns, a risk also run by NASA flagship missions outside of astrophysics. The on-orbit performance of HST and the performance expectations for JWST have also clearly established the value, scientific and otherwise, of a new and much larger space observatory covering at least the spectral region from the ultraviolet (UV) through the visible (VIS) to the near infrared (NIR). But the future great observatories will have to be built at a time when, for the foreseeable future, NASA budgets are almost certain to be flat or declining.

"ron.polidan@ngc.com; phone 1 310-812-0199; fax 1310-812-7443; northropgrumman.com

Space Telescopes and Instrumentation 2014: Optical, Infrared, and Millimeter Wave, edited by Jacobus M. Oschmann, Jr., Mark Clampin, Giovanni G. Fazio, Howard A. MacEwen, Proc. of SPIE Vol. 9143, 914319 • ( 2014 SPIE CCC code: $0277-786 \mathrm{X} / 14 / \$ 18 \cdot$ doi: $10.1117 / 12.2057161$ 
There have been a number of studies of the mission concepts and technologies for larger space observatories, identified as the Advanced Technology Large Aperture Space Telescope (ATLAST) ${ }^{1,2}$ and the Modular Assembled Space Telescope (MAST) ${ }^{3}$. These studies have established the technical feasibility of a number of sizes and designs, and the science utility will be discussed in a few paragraphs. Costing factors have been addressed in other studies ${ }^{4,5}$. The general conclusion of these studies is the fiscal incompatibility of the required large space telescope designs with the expected NASA budgets referred to in the preceding paragraph. An independent, and more detailed, analysis of this same conundrum, reaching the same conclusions regarding the incompatibility of the fiscal and the technical can be found in Arenberg et $\mathrm{al}^{6}$ in paper 9143-36 at this conference.

It is therefore essential to identify and implement a new development concept for large space telescopes that increases their affordability while, over time, providing the much improved resolution and light collecting power required to do the forefront science. It is our contention that, partly due to the annual funding cycle that NASA must employ, the root cause of the cancellation or delay risks run by a Flagship class mission is due to the annual cost of the mission. When this cost consumes too great a percentage of the yearly NASA budget it threatens all other missions, even those that are operating successfully and may offer an extended lifetime of great scientific merit. With multiple missions of all sizes threatened, the science community and NASA begin to consider draconic measures to reduce the annual cost of the Flagship mission: i.e., cancellation or program down-sizing and/or extension.

Potentially, it may be possible to overcome most of these programmatic risks by implementing some of their characteristics immediately in the initial stages of the program, effectively trading longer time to ultimate completion for greater affordability. Specifically, design, construction, and launch of the space telescope will be conducted in a number of stages, each producing a complete telescope fully capable of valuable scientific observations. Stage 1 will form the core of the observatory and will provide selected improvements upon systems operating at the planned launch date. Succeeding Stages will build upon the Stage 1 observatory in several year increments (nominally about 5 years between launches), and will add mirrors, structures, and instruments to the Stage 1 telescope. A full Flagship capability ${ }^{\dagger}$ will be achieved by Stage 3 (details of one possible approach are provided in the Reference Concept section below). Future, even more capable Stages are, of course, possible, but are not addressed in this paper.

\section{This is the concept for the Evolvable Space Telescope (EST).}

\section{EST PROGRAMMATICS}

As noted above, the development and deployment of EST will be conducted in a number of Stages, each of increasing capability, culminating in the full capability of an ATLAST observatory. Programmatically, this strategy will succeed if and only if the annual budget for each Stage is constrained below a reasonable fraction of the NASA astrophysics budget, and that fraction is well known to the astrophysics community. Thus, the EST programmatic strategy is, to a large extent, a design-to-annual-cost strategy combined with a "science-as-you go" strategy that is coupled with the requirements established by the principal science drivers. The annual cost target, still to be developed, will need to be based upon two principal factors: sufficiency for EST to achieve scientific viability with early flight stages so that the community will have continuing access to essential observational data, but low enough in cost to enable significant progress in astrophysics missions in other science regions.

The program will be structured with a modest cost, extended development in mind, enabling steady progress while capitalizing upon economies of scale as possible. Perhaps most importantly, maintaining a conservative but steady expenditure rate will protect EST from the effects of downsizing, which include inefficient use of personnel and equipment and increased expenditures in later program stages. As an illustrative example, if all three Stages can be acquired and deployed within a planned period of 15 years and the annual spend rate maintained at approximately $\$ 400 \mathrm{M}$ (current year dollars), a full ATLAST capability will be deployed for a total cost of $\sim \$ 8 \mathrm{~B}$. Assuming that the

\footnotetext{
${ }^{\dagger}$ Fully equivalent to the largest version (16 - 20 meter aperture) of the Advanced Technology Large Aperture Space Telescope (ATLAST).

${ }^{\ddagger}$ At a level to be determined.
} 
total available for NASA astrophysics remains at about $\$ 1,200 \mathrm{M}$ as it has for most of the past decade, this will still leave financial room for other, extensive astrophysics systems development.

It should also be noted that the only costs that will be addressed in the proposed EST study will be those to be financed directly and managed by NASA. This rule is intended primarily to reduce program turbulence over its long lifetime. Other organizations, national or international, public or private, may be able to make major contributions to EST once it enters development, but a central management will be essential to maintaining the viability of the program.

\section{SCIENCE DRIVERS}

A fundamental design rule for EST will be the requirement that each Stage, beginning with Stage 1 itself, will be designed to contribute significant science beyond that which constitutes the then current state-of-knowledge in important sub-fields of astronomy. The specifics of this requirement will be detailed in the design studies for the separate Stages but, as a minimum, will be based upon the science drivers used in the ATLAST studies by Green, Postman and others $^{1,7,8,9}$ and a few examples are shown in Figure 1. It will be necessary to derive intermediate science requirements that contribute to the attainment of these goals using the lesser capabilities of the intermediate stages of EST.

These science drivers, at all levels of the EST program, will address multiple questions, many of which are organized around three topics which are paraphrased here and may be reviewed in detail in the cited references for ATLAST and MAST.

- Exoplanet characterization and the discovery of life (specifically, spectral biomarkers) outside the solar system. Characterization of statistically significant numbers of suspected life-bearing planets must await observations of larger numbers of candidate planets by the later stages of EST. However, this characterization can begin for a few of the nearest systems with EST Stage 1 , a confirmed biomarker on even a single planet will be a foundational validation for astrobiology.

- The origin and evolution of the universe, including star formation and galaxy assembly, as based upon stellar population histories. The stellar populations that can be sampled by the early stages of EST are not believed to be sufficiently representative of the galactic population as a whole, but refinement of the differences may provide insight into their causes, providing different evidence regarding the evolution of the universe, particularly its later stages.

- Distribution and the nature of dark energy and dark matter, which is determined largely through measurement of the transverse proper motions of neighboring dwarf spheroidal galaxies because of the poor angular resolution of existing observatories. Definitive measurements will only become possible with the later stages of EST, but early Stages may provide sufficient evidence to begin narrowing the range of possibilities at an early date.

Other topics, such as solar system physics (planetary and small body) may be addressed by EST as well. Indeed, EST can be expected to follow the path of almost all early astronomical observatories, uncovering and resolving new issues that cannot be foreseen at this time and generating needs for more and different observations from still newer observatories over time.
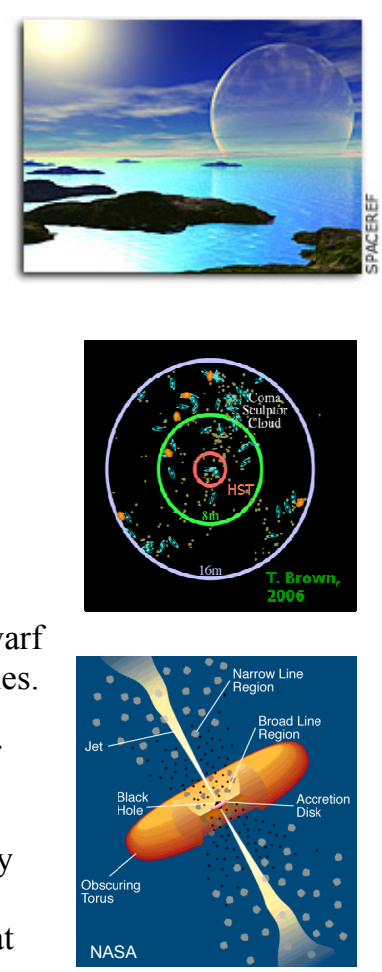

Figure 1: Example Science programs for EST

\section{MISSION CONCEPT}

The preceding introductory sections defined the Evolvable Space Telescope (EST), outlined its value, rationale, and timeliness (scientific and otherwise), and sketched the approach and expected products of the feasibility study needed as the first step towards an EST. This section will outline important aspects of the programmatic approach and 
environment, while later sections will present more details of the trade space accessible to the general concept, outline near term studies needed to begin assessment of the concept, provide an example of a generic design to suggest the sort of detailed study that will be needed, and describe the in-space servicing capabilities and technologies expected to be available at least through Stage 1 of EST.

\subsection{Orbit}

EST is envisioned as a Sun-Earth $\mathrm{L}_{2}$ (SEL2) based observatory. This location offers an excellent observation environment, will be well understood from years of JWST performance at SEL2, and has relatively low delta-V requirements if we need to move the observatory to a convenient nearer Earth location or easier access from Earth than other possible orbits, if the servicing is to be done at SEL2.

\subsection{Measures of Merit}

As a very large space telescope, ultimately similar in capability to ATLAST, EST will be subject to a large number of measures of merit, most notably in terms of performance and affordability. These will be addressed in detail over the extended study, but there are three that particularly distinguish the EST mission concept: evolvability, adaptability, and serviceability.

- Evolvability. This, of course, is the core of the EST concept, and directly implies that there will be several generations, or Stages, of EST over the system life cycle. These Stages will be separated from each other by several years (nominally five) and will provide substantial performance advances based upon the evolution of the science drivers and/or foundational technologies. Future Stages clearly cannot be specified at this time, but might consist, for example, of increased aperture size, a new instrument suite, and the like (perhaps as much as an additional telescope to be assembled into the existing vehicle). Stage 1 will be designed to provide significant enhancements against current science drivers and will form the basis of a future large telescope (i.e., starting from a single monolithic aperture of 4 to 5 meters, it will evolve to a 16 to 20 meter aperture).

- Adaptability. Evolvability will not occur in a static environment, and EST will have to respond to unplanned changes in multiple conditions. Many are quite obvious, and the following list in no way exhausts the possibilities:

- Budgetary changes, either negative or positive, and affecting either the entire NASA budget or priorities within that budget. See additional discussion below.

- Political changes, either within the nation or geopolitical in nature.

- Technical failures or shortcomings (or, alternatively, unexpected technical successes).

- Serviceability. This measure is essential to each of the preceding two, since it directly addresses the program's ability to maintain performance against failures or wear and tear, major or minor; and the ability to enhance or upgrade systems, again including minor enhancements or Stage changes. It is directly related to the continued performance of EST over many decades, lasting perhaps as long as 50 or more years.

\subsection{Budget Factors}

As noted in the previous section, it will be essential for EST to be adaptable under changing budget conditions, since the budget environment is and will remain highly volatile and adjustable on a short-term basis (generally annually). The study will employ several assumptions:

- Only NASA costs will be addressed in our analysis, although we acknowledge that additional interest and support could come from other government agencies.

- Reduction of JWST development costs will begin in FY 2017, but those resources will be assumed by the WFIRST Program.

- Costs must be adjusted for inflation to provide a constant basis of comparison, and an explicit basis of estimate provided.

- Approximately \$2-3B USD (current year dollars) will be used to Stage 1 development and launch, with the remaining funding spread over the two succeeding Stages. 
In addition, a small number of budgetary requirements will be imposed on the output of the study to control the affordability and therefore the feasibility of EST:

- The budget profile must remain essentially flat, thus implying that funding for each Stage will begin to ramp up as that for the preceding Stage is reduced.

- The total cost for the first three Stages will not exceed that of a single development for a Flagship mission of equal capability.

\subsection{Launch Cadence}

Based upon a 5 meter interior diameter launch shroud, the first Stage is baselined to employ a $\sim 4$ meter unit cell (flat-toflat hexagon). The unit cell can be larger if a larger shroud is available, or smaller if budget conditions worsen. There are several families of possible launch vehicles: ULA (principally Atlas V and Delta IV), the Space-X Falcon 9, and the Ariane 5, for example.

The baseline program will consist of Stages 2 and 3 to build on the Stage 1 telescope. These have not as yet been designed, but the goal is a final Stage 3 aperture in the 16 to 20 meter range, with launches occurring at approximately 5year intervals. Stages beyond 3 are possible, but early concept development will not actively pursue these levels, but will take no actions that will preclude missions as long as 50 to 60 years.

\subsection{Instrument Suites}

EST instruments, beginning with Stage 1, will be mounted in a manner that will enable either a simple replacement of the entire suite or individual instrument replacement. By the time EST is launched we are assuming that the ongoing revolution in astrophysics instruments will continue, particularly in the area of Integral Field Spectrographs and other similar imagery/spectroscopy instruments, and that it is likely that this type of instrument would comprise the "core" general use instrument for EST. In addition we would expect specialized instruments such as starlight suppression systems (starshades or coronagraphs - EST will be starshade compatible) and likely other focused application instruments for specific science goals. These instruments would be selected through a Science Mission Directorate (SMD) competitive process based upon both the prioritized science drivers and the technological maturity of the relevant instruments at the time of the mission. For the purpose of this study we are simply assuming that there will be a core instrument suite for EST with ancillary "specialized" instruments.

\subsection{Outreach}

Just as with the HST and JWST, EST will be a key element in NASA astrophysics outreach, both directly to the public and educational establishments and to senior government officials. It will be the prominent space telescope that is most readily understood by all members of the public, and will therefore provide key support for other science missions. They will watch it be launched and evolve, and watch the science return become ever more spectacular.

Since EST, and some of its possible support elements, can provide significant support to both human exploration and planetary science missions, it will be essential to establish a firm working relationship at least with the Planetary Sciences Division of SMD and with the Human Exploration and Operations Mission Directorate (HEOMD).

\section{EST TRADE SPACE}

The preliminary concept study will need to make a large number of technical tradeoffs in order to define a basic reference design for future in-depth study. Although a totally complete set cannot be established in the early stages, these tradeoffs will necessarily include those listed in the following table. Some tradeoffs that would be included in a totally bottoms-up study have already been made, and their results are included in the assumptions. Two of these are:

- The most significant and obvious is the selection of a SEL2 halo orbit as the location for EST operation, which was based upon tradeoff studies for several other systems and upon the low transfer energy required for effective servicing of the telescope. 
- The operating band (UVOIR) is a second major, completed tradeoff, based largely upon its historical role in the astronomical sciences. However, the EST concept is not limited to this waveband, so other bands would also be possible (i.e., IR with the addition of instrument cryocooling).

Note that this table does not address tradeoffs among instruments, which will have to be considered in later work once the telescope (and spacecraft) designs are more settled for each Stage. These analyses will be largely based upon detailed considerations of the primary science drivers, with allowances made for the flexibility needed to address unexpected scientific questions and opportunities.

\begin{tabular}{|l|l|}
\hline \multicolumn{1}{|c|}{ GENERAL TOPIC } & \multicolumn{1}{c|}{ SPECIFIC TOPICS / COMMENTS } \\
\hline System Basics & $\begin{array}{l}\text { Apertures for each Stage } \\
\text { Available assembly and servicing options } \\
\text { Materials, both optical and structural }\end{array}$ \\
\hline Telescope Basics & $\begin{array}{l}\text { Basic optical design } \\
\text { Segment size for each Stage }\end{array}$ \\
\hline Wavefront Control & $\begin{array}{l}\text { Distribution among telescope and instrument components } \\
\text { Passive / active elements }\end{array}$ \\
\hline Performance Options & $\begin{array}{l}\text { Maximum optimization for each Stage } \\
\text { Accept sub-optimization for individual Stages for end-to-end design }\end{array}$ \\
\hline Thermal/Light Control & $\begin{array}{l}\text { Sunshield design (optical barrel, parasol, etc.) } \\
\text { Temperature control options }\end{array}$ \\
\hline System Fault Options & $\begin{array}{l}\text { Extent of redundancy } \\
\text { Certification requirements }\end{array}$ \\
\hline $\begin{array}{l}\text { In-Space Assembly } \\
\text { Approach }\end{array}$ & $\begin{array}{l}\text { Partial or complete deployment vice assembly } \\
\text { Location(s) for assembly } \\
\text { Robotic or human presence } \\
\text { Proximity operations technologies and approaches }\end{array}$ \\
\hline
\end{tabular}

\section{THE INITIAL EST STUDY}

As with any study of a new mission concept, the first step must be to decide precisely what to study in detail and how to proceed with those detailed assessments. There is a natural flow down, possibly involving one or more iterative cycles as later steps encounter flaws in earlier conclusions that this study will follow.

- Begin by defining realistic architectural approaches for the EST, and specify their components and characteristics in detail sufficient for inter-comparison among them. This should lead to identification of a small set of possible architectures with differences significant enough to enable comparative evaluation.

- Converge on a set of technical attributes and characteristics, with associated rationales, that may be used as the basis for a priority ranking of the EST architectures. Identify the problems and issues within this set that need to be resolved, and identify the technologies (at any TRL) that could help realize EST as an operational system.

- Define a more detailed plan of action, including refinement of the achievable science needs, a more detailed definition of the observational mission as a function of time and EST Stage, and a roadmap for the maturation of the required technologies. 
As the EST Mission Concept Study develops, it should provide a source of information and insight that will help to both build community interest (including the scientific, government, and public communities) and as a construct for all of these communities to discuss, debate, and refine. Much of the discussion can be expected to revolve around evolving Design Reference Concepts. A simplified, preliminary concept is outlined in the following section.

\section{DESIGN REFERENCE CONCEPT}

This paper is not intended to provide an extensive list of design concepts that could be used to implement the Evolvable Space Telescope architectural philosophy. However, the authors believe that comprehension of this design philosophy will be assisted through discussion of at least one elementary example as a reference.

The details of the assembly of the EST Primary Mirror Assembly (PMA) are represented in Figure 2. For comparative purposes, this paper assumes that all the hexagonal segments shown are $\sim 4$ meters flat-to-flat, or approximately 4.5 meters point-to-point, although these dimensions are purely illustrative. The EST approach can accommodate any segment size, but the telescope aperture at each stage will of course scale with the segment size.

Figure 2 is intended to represent only the underlying approach to be employed in EST, and not a specific implementation of that approach. The details of possible implementations will be worked out in detailed studies to follow this initial work, but some specifics are included to provide a relatively intuitive insight into the EST concept.

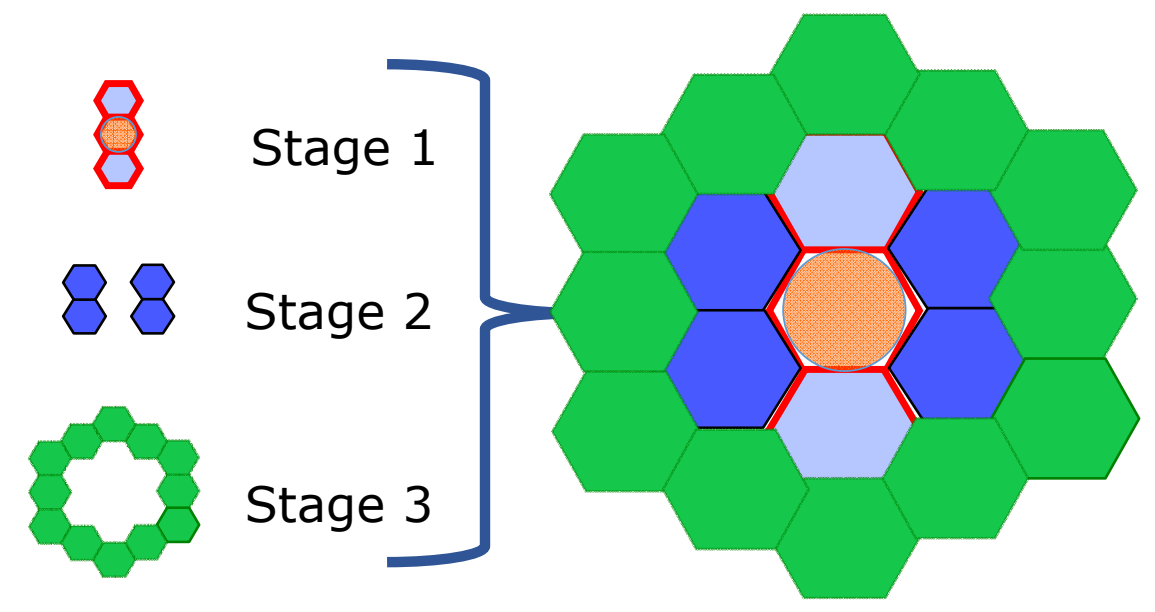

Figure 2: EST Reference Concept. See paragraph below for details.

This discussion is based upon Figure 2, which represents one possible configuration of the primary mirror in each of the three Stages:

- Stage 1. The two grey hexagons and the central circle. The hexagons form the initial PMA, and will typically be formed to the master prescription. The central circle represents the secondary mirror, and will be designed to serve the same role throughout Stages 1,2, and 3. These elements will all be orbited and assembled using the first EST launch, providing an asymmetric aperture on the order of $12 \mathrm{~m}$ by $4.5 \mathrm{~m}$.

- Stage 2. At the chosen time (about half a decade following Stage 1), four additional segments (shown in dark blue) will be launched, along with other components (e.g., new instruments) to form the Stage $212 \mathrm{~m}$ filled aperture, probably in a Cassegrain configuration.

- Stage 3. Again, approximately a half-decade following the preceding launch, the next stage of telescope evolution will occur, in this case adding 12 mirror segments (in green) to form a $20 \mathrm{~m}$ aperture for the telescope, again probably in a Cassegrain configuration. In this case, since the largest dimension of the PMA will be 
significantly increased, the upgrade will include at least a new sunshield plus other replacement components and servicing as needed.

- Stage 4 +. Later stages of EST remain possibilities for examination in the future.

\section{SERVICING CONSIDERATIONS}

As implied under the discussion of the principle measures of merit in the Mission Concept section, serviceability will be a key to the success of the Evolvable Space Telescope, particularly given the extended operational lifetime expected of the observatory. Two principal technologies are developing rapidly that will enable this critical capability: robotics/telerobotics and multiple space platforms that can form the basis for servicing operations of all types.

\subsection{Robotics/Telerobotics}

The capability of human/machine and autonomous machine systems to perform a wide variety of mechanical functions is one of the most conspicuous features of current technology. This is true in space as well as within the biosphere, despite the arduous conditions faced there, as most visibly demonstrated at the International Space Station (ISS) and on the surface of Mars, among others. These capabilities provide the foundation needed to enable the assembly and servicing of systems such as EST, regardless of their specific locations. Performance of these functions can be expected to become increasingly the responsibility of autonomous robots but, with the availability of an increasing variety of manned systems, human presence will continue to provide support both to reduce temporal latency and to assume control when unforeseeable circumstances arise.

\subsection{Service Platforms}

Implementation of an effective in-space infrastructure is in its early stages, but is proceeding with increasing rapidity, driven by the ISS and the variety of deep space missions being planned and evaluated. Systems range in size from the ISS down to electronic chip size, and are in all stages of development. A complete discussion is well out of scope of this paper, but a few relevant points are outlined in the following:

- ISS. This is the major representative of large space structures, and has a wide range of uses, such as the following:

- Experiments with assembly and servicing technologies under continuous human control.

- Assembly of "modEST " - a subscale demonstration model of EST Stages 1 and 2 that is currently being explored

- Partial assembly and providing an en route parts and consumables depot for deep space systems

- Since limited to low earth orbit, final assembly of deep space vehicles is unlikely.

- Deep Space Habitat (DSH). An inhabitable vehicle for use in deep space, for example at the Earth-Moon Libration points EML1 and EML2 (Earth-Moon $\mathrm{L}_{1}$ and $\mathrm{L}_{2}$ ). A nominal crew of four would be present during assembly and servicing operations.

- An early version could be created from an Orion capsule and a service module.

- Support major assembly and servicing tasks in deep space, with low latency times.

- Provides a (partial) prototype for a Deep Space Transport (DST) for, for example, Martian or asteroid expeditions.

- Mini Service Vehicle (MiniServ). An unmanned vehicle massing about $1000 \mathrm{~kg}$, based at EML1 or EML2 and equipped with an array of tools, manipulators, and parts.

$\circ$ Specific assembly and servicing tasks, minor to major, performed either at its basing location or at the location of a deep space mission vehicle (likely to be SEL2 for EST).

- Provide assistance to DSH servicing when needed, possibly eliminating the need for human EVAs.

- Provide unplanned or emergency services, such as replacing instruments or reaction wheels, repairing leaks and replenishing consumables, etc.

- Micro Service Vehicle (SPHERES, CUBESAT).

○ Subsidiary roles, such as inspections and service monitoring, temporary leak repairs, etc.

- Requires transport to work site.

- Nano Service Vehicle (ChipSat). Highly specialized due to small size.

- Internal repairs, such as electronic chip replacement. 
- Cover micrometeoroid gaps in sunshields.

- Also requires transport to work site.

\section{SUMMARY}

\subsection{Summary of Specific Observations}

In the process of developing this paper, several specific conclusions have become apparent to the authors. Some of these are presented in the body of the text, but they are summarized in the following:

- Trades and optimizations must be conducted at the full mission level to ensure a viable system. For example, pressures from manufacturing technologies (e.g., for different mirror segment sizes) can generate an expanding set of issues leading to sub-optimized mirror architectures.

- Servicing of the telescope, both for maintenance and repair and for designed upgrades,

- Larger mirror segment sizes (particularly > 2.5 meters) appear to simplify the PMA architectures by reducing the required number of interfaces.

- A fully passive telescope system will not work. Some combination of passive and active elements will be essential.

- The only operational orbit that makes sense will be a halo orbit about the Sun-Earth Libration point $\mathrm{L}_{2}$ : SEL2.

\subsection{Continuing Activities}

The principal remaining activity for 2014 will be to finish (by November) the mission concept study reported in this paper. In particular, by the completion of this phase of the study, we plan to have:

- Defined architectural approaches for EST.

- Converged on a set of initial technical attributes and characteristics, associating them with appropriate rationales.

- Identified the studies (mission analyses, technology needs and/or applications, etc.) that will need to be addressed during 2015

- Developed an initial technology roadmap, continuing its development into 2015 and beyond that, if executed successfully, would enable operational realization of an Evolvable Space Telescope

\subsection{General Summary}

The study team has developed a preliminary concept for an Evolvable Space Telescope (EST) architecture that can enable the development and operation of very large (> 14 meters) space observatories in an era of flat budgets. The architecture is characterized by:

- Beginning small and growing in stages to the large aperture needed to answer future science questions.

- Scheduling the build-up from smaller sizes by flexible responses to budget realities, developing science needs, and technology advances.

- Maintaining a continuous flow of science data from initial system commissioning with only short gaps for servicing (as was typical of HST).

\section{ACKNOWLEDGEMENTS}

The authors would like to acknowledge strong support and internal funding from Northrop Grumman Aerospace Systems and very helpful comments, suggestions, and criticisms from a variety of people, including Jonathan Arenberg, Alberto Conti, Lee Feinberg, Marc Postman, Theodore Swanson, and Harley Thronson. 


\section{REFERENCES}

[1] National Aeronautics and Space Administration, "ATLAST Mission Concept Study", http://www.stsci.edu/institute/atlast/atlast-mission-concept-study, NASA, Washington, DC (2009).

[2] National Aeronautics and Space Administration," "Technology Development Plan for the ATLAST", http://www.stsci.edu/institute/atlast/documents/ATLAST_Tech_Dev_Plan.pdf, NASA, Washington, DC (2009)

[3] Feinberg, L., et al, "Modular Assembled Space Telescope," Optical Engineering, 52 (9) 091803-01, SPIE (2013).

[4] Stahl, H. P., et al; "Preliminary cost model for space telescopes", Proc. SPIE. 7436, San Diego, CA, 2009

[5] Stahl, H. P., et al; "Update on multi-variable parametric cost models for ground and space telescopes", Proc. SPIE, 8442, Amsterdam, Netherlands, 2012.

[6] Arenberg, J., et al, "A new paradigm for space astrophysics mission design”, Proc. SPIE 9143, Montreal, QC, Canada (2014), In press.

[7] Green, J. et al. 2008, “The Modern Universe Space Telescope,” NASA Space Science Vision Missions, 224, 229.

[8] Postman, M. et al., "Science drivers and requirements for an Advanced Technology Large Aperture Space Telescope (ATLAST): Implications for technology development and synergies with other future facilities", Proc. SPIE, 7731 (2010) (arXiv:1008.1942)

[9] Postman, M. et al., "Advanced Technology Large Aperture Space Telescope; science drivers and technology developments", Optical Engineering, 51(1), 011007 (January 2012) 